\title{
Collaborative systems for telemedicine diagnosis accuracy
}

\author{
Jacques Tene Koyazo ${ }^{1}$, Moise Avoci Ugwiri ${ }^{2}$, Aimé Lay-Ekuakille ${ }^{3}$, Maria Fazio ${ }^{1}$, Massimo Villari $^{1}$, \\ Consolatina Liguori ${ }^{2}$ \\ 1 Department of Mathematics and computer science, Physical sciences and Earth science, University of Messina, Lecce 73100, Italy \\ 2 Department of Industrial Engineering, University of Salerno, Fisciano 84084, Italy \\ ${ }^{3}$ Department of Innovation Engineering, University of Salento, Lecce 73100, Italy
}

\begin{abstract}
The transmission of medical data and the possibility for distant healthcare structures to share experiments about a given medical case raises several conceptual and technical questions. Good remote healthcare monitoring deals with more problems in personalized heath data processing compared to the traditional methods nowadays used in several parts of hospitals in the world. The adoption of telemedicine in the healthcare sector has significantly changed medical collaboration. However, to provide good telemedicine services through new technologies such as cloud computing, cloud storage, and so on, a suitable and adaptable framework should be designed. Moreover, in the chain of medical information exchange, between requesting agencies, including physicians, a secure and collaborative platform enhanced the decision-making process. This paper provides an in-depth literature review on the interaction that telemedicine has with cloud-based computing. On the other hand, the paper proposes a framework that can allow various research organizations, healthcare sectors, and government agencies to log data, develop collaborative analysis, and support decision-making. The electrocardiogram (ECG) and electroencephalogram EEG case studies demonstrate the benefit of the proposed approach in data reduction and high-fidelity signal processing to a local level; this can make possible the extracted characteristic features to be communicated to the cloud database.
\end{abstract}

\section{Section: RESEARCH PAPER}

Keywords: Signal processing; biomedical; collaborative edge; cloud computing, accuracy; theranostics; measurement

Citation: Jacques Tene Koyazo, Moise Avoci Ugwiri, Aimé Lay-Ekuakille, Maria Fazio, Massimo Villari, Consolatina Liguori, Collaborative systems for telemedicine diagnosis accuracy, Acta IMEKO, vol. 10, no. 3, article 26, September 2021, identifier: IMEKO-ACTA-10 (2021)-03-26

Section Editor: Francesco Lamonaca, University of Calabria, Italy

Received June 12, 2021; In final form August 5, 2021; Published September 2021

Corresponding author: Jacques Tene Koyazo, email: jacquestene2013@gmail.com

\section{INTRODUCTION}

The spectacular progress in communication technology has boosted the telemedicine which seems to be a new medical practice preference in medical areas [1]. One of the benefits carried out by this new medical discipline is, for instance, the stomatological diagnosis. Thanks to the communication and computer technology, the stomatological diagnosis can provide a safe and reliable remote diagnostic, counselling care, distance education and other information services of medical activities [2]. In recent years, various standards for health care research regulation have been developed. Evidences in the literature have proven that studies based on IEEE and ISO standards [3] enable a good compliance in terms of collaboration with healthcare industries, government agencies and research institutes towards developing novel approaches and methods for handling and controlling diseases. In fact, implementing a reliable collaboration system in telemedicine has a tremendous advantage. It breaks distance restrictions, so that different medical institutions can provide diagnosis; it improves the exchange accuracy for medical advices; it provides a cooperative working environment suitable to share data and information helping in dealing with emergencies. Since then, telemedecine has demonstrated a huge prospect thanks to everyday development of information and telecommunication technology [4]. A part from pravicy and security concerns, medical data transmission still face data transmission problem. According to statistics provided by the second Xiangya hospital [5], most of medical data produce over $1 \mathrm{~GB}$, and those massive generated data often tend to have a growth rate exceeding the speed of the expansion of the mobile IoT bandwidth. This aspect was confirmed by the Cisco's yearbook report [6] demonstrating that they can account for more than $85 \%$ of data traffic. This paper is elaborated on the assumption that sensors are used to collect comprehensive physiological information from targeted patients and uses cloud to store and analized those information. The data from the latter process are sent to the service provider for deep investigation. At the same time, those information can be used to remotely monitor health condition of the patient. Various sensors are nowadays used in clinical care, storing data from the used sensors in the cloud for more complex analysis and share the results with 
the medical professional for further examination is the core idea beside this paper.

\section{DIAGNOSIS IN TELEMEDECINE THROUGH A COLLABORATIVE FRAMEWORK: A LITERATURE SURVEY}

\subsection{General overview}

The Internet of Things (IoT) in collaborative medical framework is considered to be the most fundamental aspect enabling collaboration in telemedicine, because it allows healthcare applications to fully use the Io' $\mathrm{T}$ and cloud computing [7]-[9]. The framework also provides protocols to support the communication as well as broadcast of raw medical signals from different sensors and smart devices to a network of fog nodes. A good insight of a collaborative framework has been introduced by Yang et al. [10] and Almotiri et al.[11], where they have suggested an architecture able to collect data on the patient health thanks to several sensors, and transfert them to a remote server for processing and giving the possibility to display the results. The Figure 1 shows essential components needed in a collaborative framework for collaborative system in telemedicine diagnosis.

In ideal situation, sensors have to constantly collect patient's health condition and vital information. The collected data are then sent to hand-held devices via edge router where it will analyzed and store on a cloud computing platform for further evaluations as presented in the Figure 2.

It is worth to mention here that, sensors are continuously sending patient's vital signs as raw information such as electomyograpy (EMG), electrocardiogram (ECG), electroencephalogram (EEG), body temperature, blood glucose (BG) and so on. The good data exchange platform architecture ensures that all sensors operate smoothly so that users can interact with them easily. ECG and EEG are demonstrated in section 3 of this paper as show cases.

\subsection{Cloud computing for telemedicine}

In information technology, the cloud computing becomes the hottest subject nowadays. The computing ressources allocated by is in fact on-demand, scalable and secure to users. In a work done by Sultan et al. [12] cloud computing is described as the backbone of IoT health systems. The cloud computing has the advantage of providing the capability of sharing information among health professionals, research institutes and patients in a more

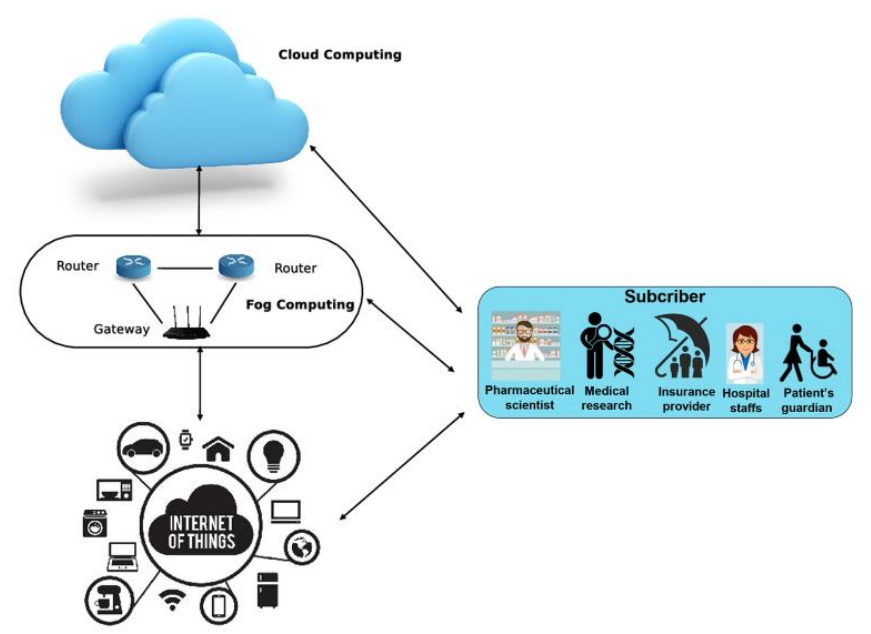

Figure 1. Basic subsets in collaborative framework for heathcare in telemedicine.

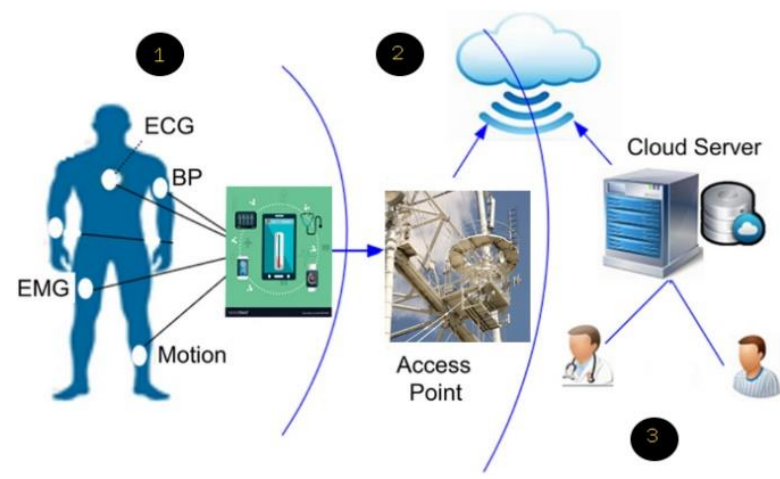

Figure 2. A typology for remote patient monitoring using body sensors.

structured and organized maner, which minimized the risks of medical record lost [13]. The Figure 3 presents the platform of a remote healthcare monitoring system based on cloud computing. Each layer in the platform is designed to handle a specific task and it can be implemented in the way to serve various query for healthcare.

The cloud storage and the multiple tenants access control are the "master layer" of the platform. It assures the collection of the healthcare data from sensors such presented in the Figure 2 (layer 1). The healthcare annotation layer solves data heterogeneity issue, which is a big deal in signal processing discipline. The fact that, sensors used for telemedicine purpose generate data of various type, this complexifies the data sharing automation among agencies. One way is to just create an open linked life data sets to annotate personal healthcare data and integrate dispersed data in a patient-centric pattern for cloud application [14]. The data analysis layer processes data stored in the cloud to assist in

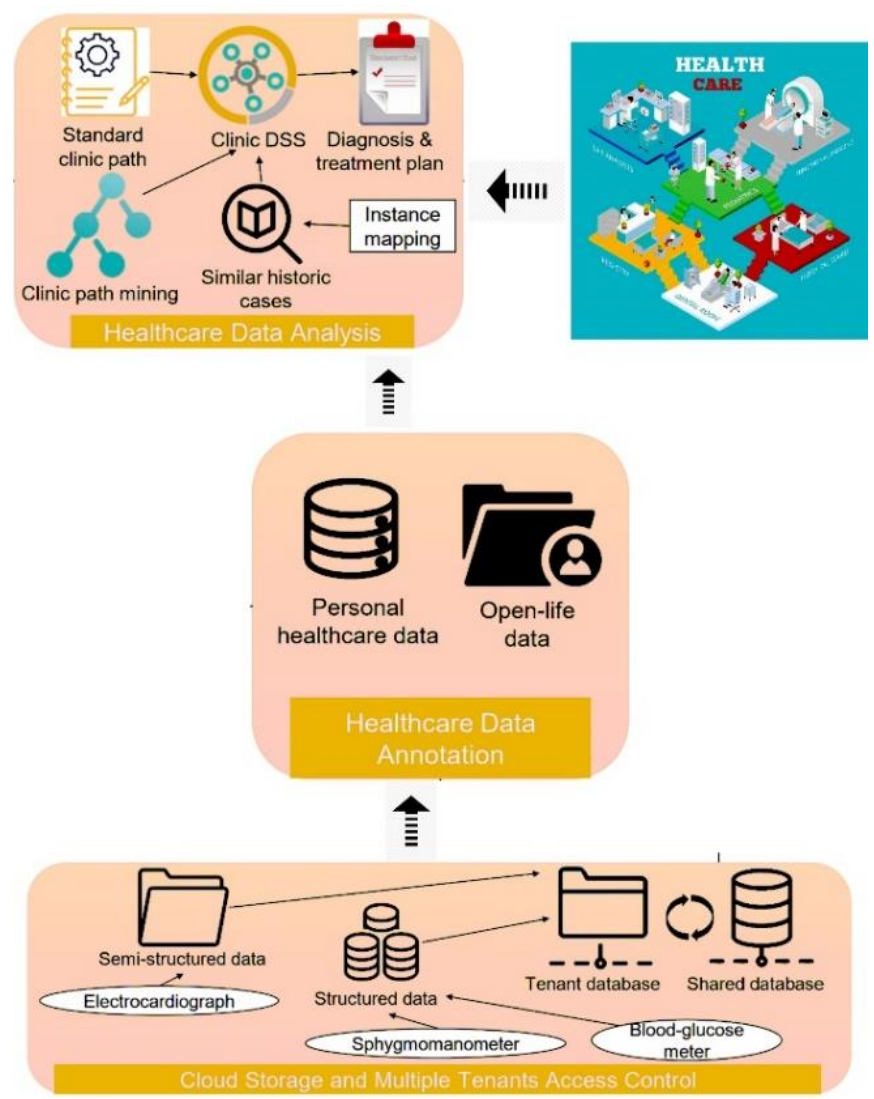

Figure 3. Cloud-computing based on remote health monitoring - functional platform. 


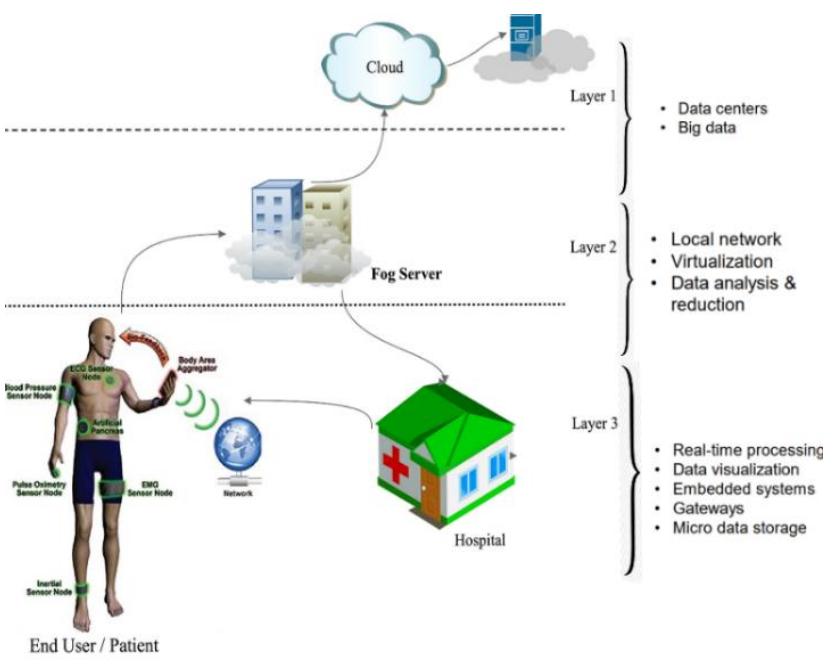

Figure 4. Main attributes in fog computing architecture : an illustration.

clinical decision making. One can see in Figure 4, mining algorithms are constructed in order to induce clinical paths from personal healthcare data.

It is important to point out here that, major part of the cloud data centers are geographically centralized and located way far from end-users [15]. For telemedicine application which often requires immediate real-time feedback, communication between users and remote cloud servers are the reason of major issues like delays in round-trip, network congestion, and so on. Those observations led to new evolutions in cloud computing, such as fog computing and big data, the cloud computing got then extended and got able to support high scalable computing platforms [16]. In [6], one can evidently see that, CISCO was the first to introduce the fog computing concept as a possible solution to extend cloud network edge computing power and storage capacity. Fog computing is known to be closer to devices and possesses a dense geographical distribution, so applications and services can be placed at the edge of the local network, which reduces bandwith usage latency. In this way cloud gets closer to the user, and the processing gets done locally, minimizing network ltency and bandwidth usage. The main fog computing architecture as illustrated in the Figure 4.

The interest beside the use of fog computing layer is deeply discussed by many authors, where they analyzed the role of fog computing in implementing healthcare monitoring framework and they proposed a mediator layer to receive raw information from sensor devices and then store them on the cloud.

\section{CASE STUDY AND DISCUSSIONS}

Embracing collaborative edge computing helps healthcare organizations visibility over patient care cycles. Sharing information practionners (phycians or health professionals) can give organizations a clear big picture view of what's going on and how to deal with it. Moreover, this technology effectively contributes to the process of organizations of the health system (hospital or health center) from the point of view of the exchange of data in a reliable way, in particular for urgent critical cases presented by patients, such as neurological, cardiological problem, etc.

Figure 5 represents the proposed collaboration architecture of two healthcare centers, respectively $\mathrm{A}$ and $\mathrm{B}$. We consider two patients under ECG and EEG diagnosis, as shown in the diagram, who are. This part constitutes the first block for the diagnosis based on the designed architecture, specifically for ECG and ECG study cases. The diagnosis is made for two patients, each in these healthcare centers where their biosignals are captured. The second considered a block of the process, and the result is composed of three compartments where each one plays a specific role, starting with the acquisition in the local interface, and the data received from another health center, via the data management, and the local storage for the final result after analysis and interpretation of data at the level of the third compartment (Data analysis).

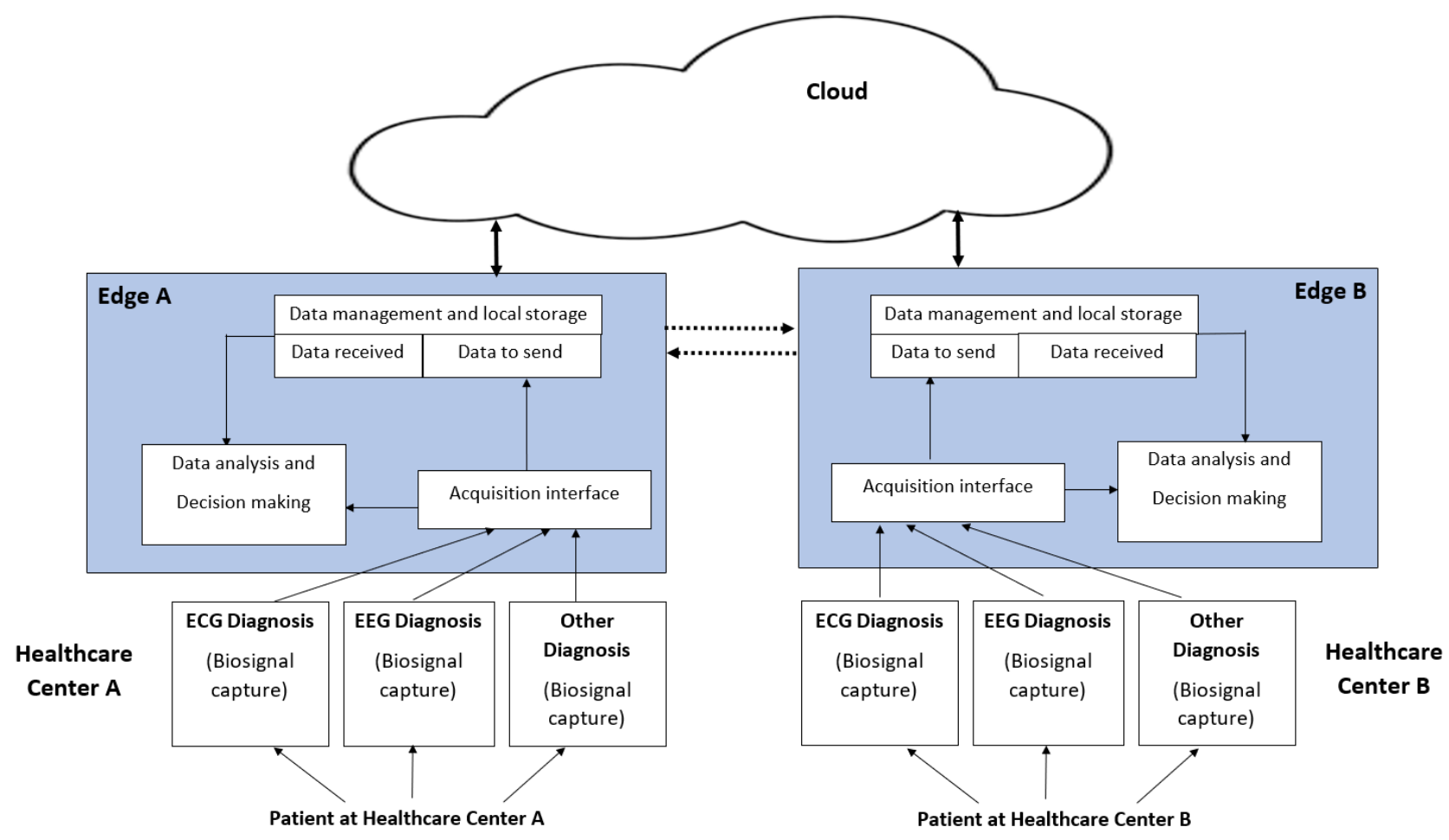

Figure 5. The proposed collaborative architecture. 
This nature of collaboration between the network edges is attributed in two healthcare centers named Edge A and Edge B to ensure the reliability, storage, and processing of geographically distributed data. One of the main scopes of this architectural configuration is the exchange of diagnosis between two healthcare centers or multiple healthcare ones. This exchange is necessary to carry out specific comparisons amongst patients of the same age range and the same characteristics. Due to heavy file dimensions, the proposed architecture also allows performing remote access without displacing files. That is possible thanks to the cloud.

\subsection{ECG Monitoring}

In medical applications, ECG sensors record the electrical activity of a heart at rest, then deliver acquired information about HR and rhythm. The recorded information is crutial in early prediction of a heart enlargement due to hypertension or heart attack. The integration of IoT in ECG for telemedicine practices has a tremendous benefits and high potential to warn users about heart rate abnormality, which is a vital sign of early heart desease detection. In this paper, two patients have been considered. In the Figure 6, the output ECG signal of two patients is presented, where the filtering sequence uses Pan Tompkin's QRS detector. For the comparison purpose, the signals have been downsampled to $250 \mathrm{~Hz}$. Each window represents 5.5 seconds of data. Each stage introduces a delay with a cumulative delay of 40 samples.

For a optimum filtering, the butterworth filter is used in this study. The filtering range is between $4 \mathrm{~Hz}$ and $20 \mathrm{~Hz}$, the filter is of order 4 . The proposed processing algorithm is implemented in matlab.

In Figure 7, one can see the respiratory rate which is a critical and one of the vital signs used in telemedicine. R-R interval has been used for detection. Due to the change in heart rate synchronized with respiration, the R-R interval of the ECG is short during the respiration, and long during expiration [18]. However, the morphology of a heart can vary greatly depending on the patient as shown in Figure 7. Usually, normal heartbeat for a patient can resemble an abnormal beat for another. The Hamilton and Tompkins algorithms used in this paper [19] are then used for peak energy amplitude detection rather than the detailed morphology of the ECG.

\subsection{EEG monitoring}

EEG are known to be interesting source of information for remote healthcare and can be associate to ECG for enhancing the diagnosis task [12]. The issue though is to apply appropriate techniques to extract information prior to upload to the cloud, so that medical agencies can take advantage of them for proper and accurate diagnosis. This paper adopted the Filter Diagonalization Methods exploited by A. Lay-Ekuakille et al. [20] to extract relevant parameters such as complex frequencies from a given window. EEG can be seen as signals that are sums of damped exponentials, which makes suitable to apply FDM and/or the Decimeted Signal Diagonalization (DSD). The bandlimited decimated signal can be modeled as

$$
C_{n}^{\text {bld }}=\sum_{k=1}^{K} d_{k} \mathrm{e}^{-\mathrm{j} \omega n \tau_{D}}, \quad \operatorname{Im} \omega_{k}<0,
$$

where $\omega_{k}$ and $d_{k}$ are complex frequencies and amplitudes respectively. If $M$ is the times at which the signal is sampled, then for each of the $M$ signals, the diagnonalization for FDM algorithm can be implemented as follow:

$$
\begin{aligned}
& C_{n}^{\text {bld }}=\sum_{k=1}^{K} d_{k} \mathrm{e}^{-\mathrm{j} \omega n \tau_{D}} \Rightarrow U_{1} B_{1 k}=u_{1 k} U_{0} B_{1 k} \\
& C_{2 n}^{\text {bld }}=\sum_{k=1}^{K} d_{2 k} \mathrm{e}^{-\mathrm{j} \omega n \tau_{D} \Rightarrow U_{1} B_{2 k}=u_{2 k} U_{0} B_{2 k} .}
\end{aligned}
$$
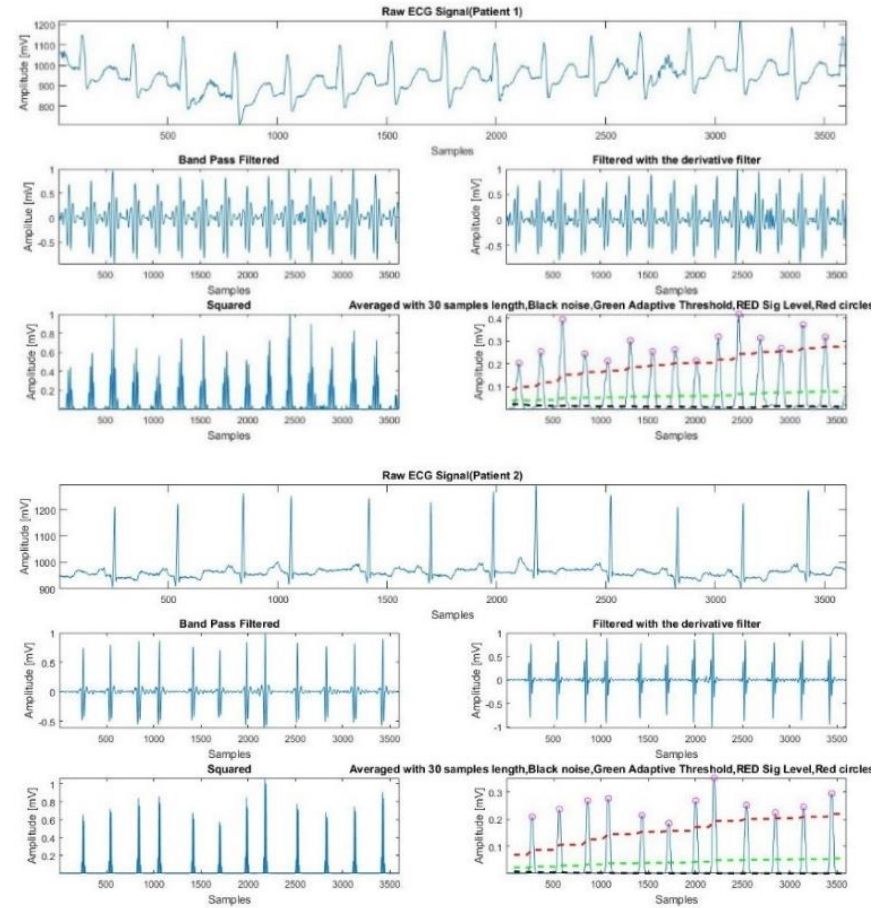

Figure 6. Processing sequences for patient 1 and patient 2 .
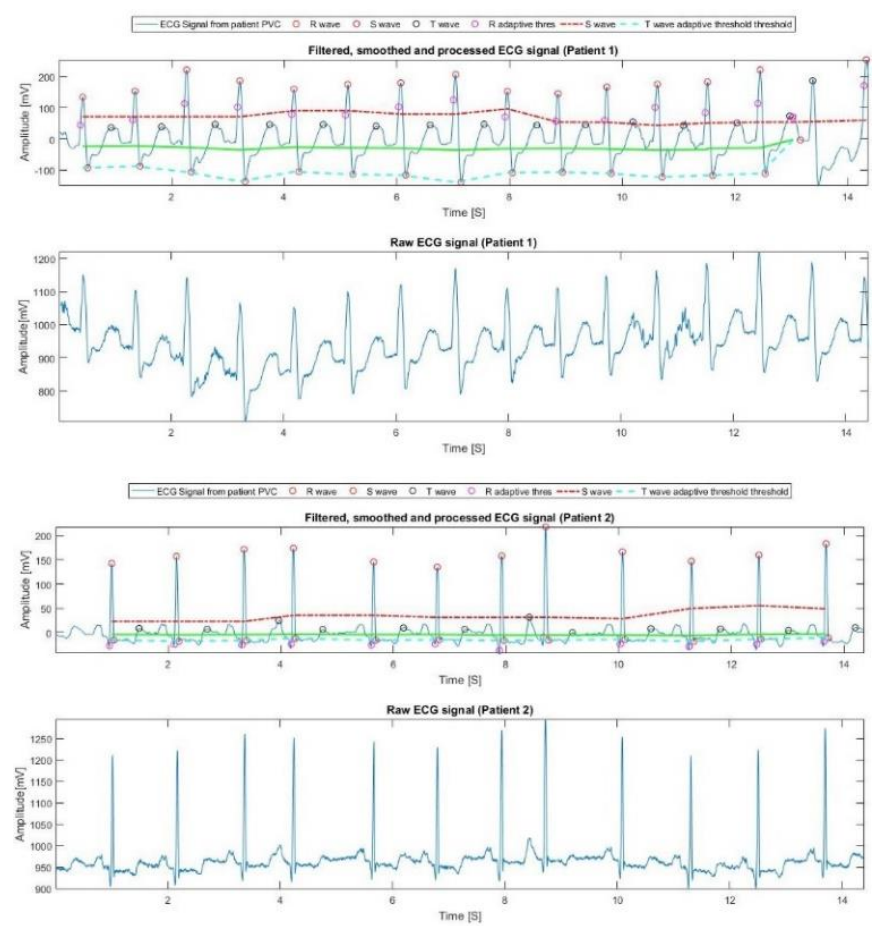

Figure 7. Filtered, smoothed and processed ECG for patient 1 and patient 2. 
In (2) and (3), complex frequencies are extracted from the eigenvalues $u_{1 k}$ and $u_{2 k}$. The reader is encouraged to find more detailed on FDM algorithm structure in [21]. As for the ECG case, the EEG is considered for the patient 1 and patient 2, where patient 1 is the unsuspected child and the latter is the suspected child. Figure 8 presents the bisepstrum of the signal ranging from 10801 up to 12000 samples. The bisepstrum is built for the second interval for the patient 2 as can be seen in Figure 9.

An important clinical feature for both suspected and unsuspected cases (epilepsy in this case) are presented in Figure 10 and Figure 11 respectively for patient 1 and patient 2.

As stated above, cloud computing provides secure platform for two-way sharing of research data across different agencies or institutions. Platforms such as google-cloud, GIFT-cloud, etc... are designed to meet the need of collaborative research project by simplifying data transfer. The EEG and ECG characteristic features obtained in this elaboration, make easy to integrate with IT local infrastructure of the institutions that provided clinical data and expertise, with end-user within routine clinical workflow. The results are presented such as supports for varied collaboration agreements between institutions and related access control restrictions. The improved scheme proposed in Figure 5 , makes possible the configuration as well as the update, and can allow new modalities to be added via the server without requiring software updates. Features extracted such as bispectrum presented in Figure 12 simplified the development of the research software. The idea is straitforward, such that the development allows to automatically fetch the data directely from the server, given the fact data has been uploaded from research center (in our case). These features are not just accurate, but also useful for both medical research projects where data
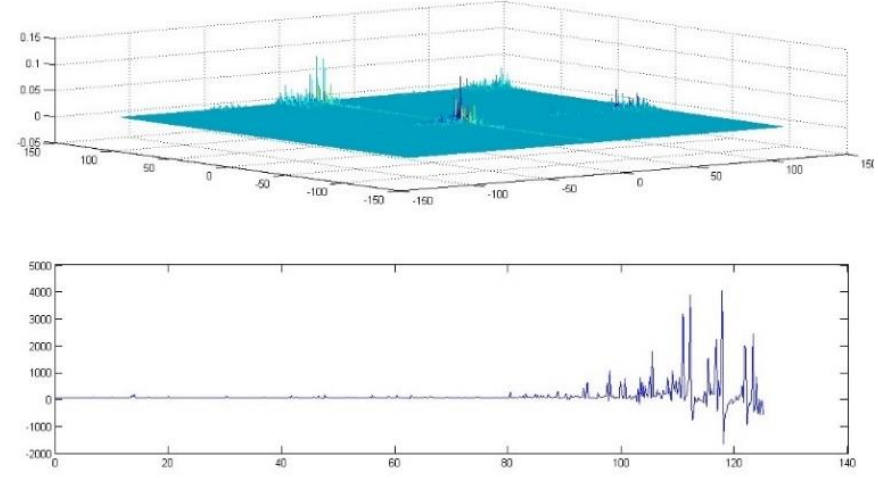

Figure 8. The bispectrum and associate representation for patient 1
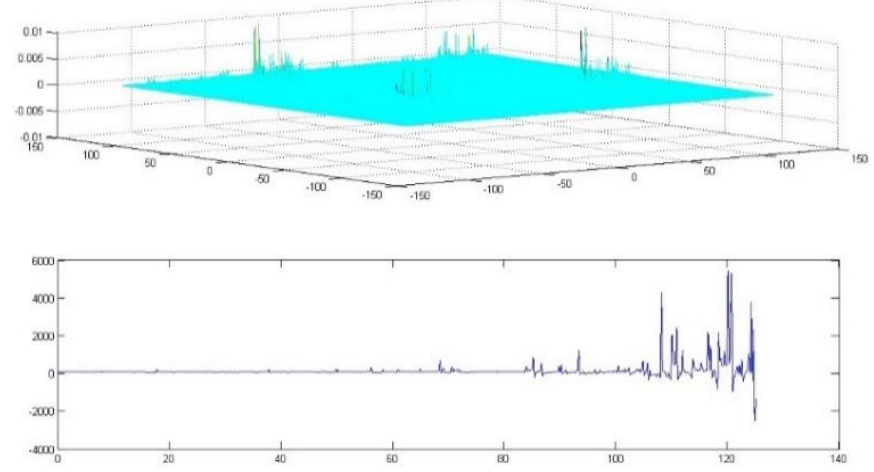

Figure 9. The bispectrum and associate representation for patient 2 (here the sample is $7201-8400$ ). sharing is required between researchers and clinical institutions and medical professionals for decision making.

\section{CONCLUSIONS}

Telemedicine aimed to provide high-quality medical services, given that healthcare facilities nowadays hardly satisfy populations' needs due to limitations of public medical resources and infrastructures. This research proposed a cloud-computingbased architecture for decentralized and collaborative diagnosis by highlighting patients' data storage after meaningful feature extraction. In this way, a medical professional can rapidly grasp the patient's state in question and make an accurate decision easily. As reported at the beginning of section 3, collaborative edge computing certainly helps healthcare organizations in the spirit of connecting hardware and software issues in a unique platform for decision making in the interest of patients [22], [23].

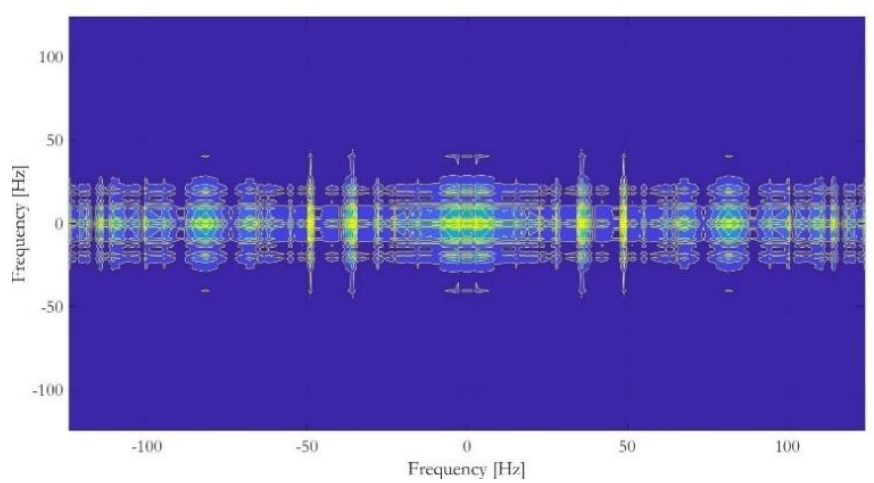

Figure 10. The bispectrum and associate representation for patient 1 (here the sample is 7201 - 8400).

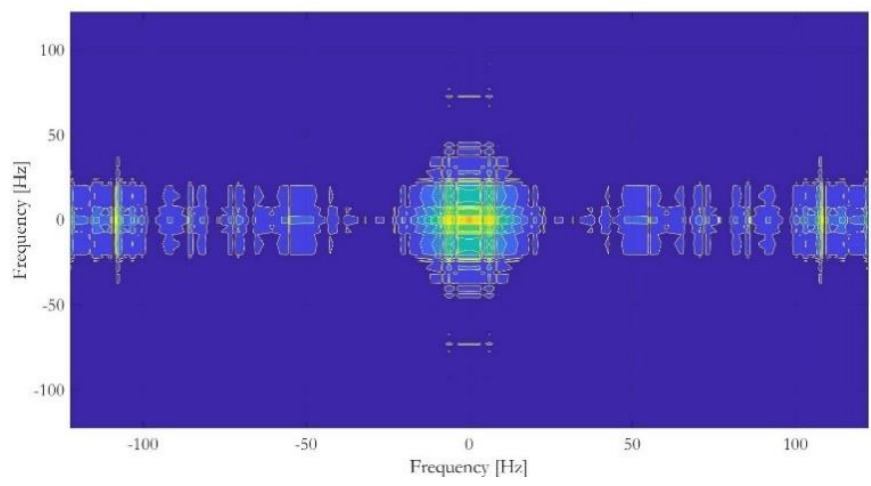

Figure 11. The bispectrum and associate representation for patient 2 (here the sample is $7201-8400$ ).

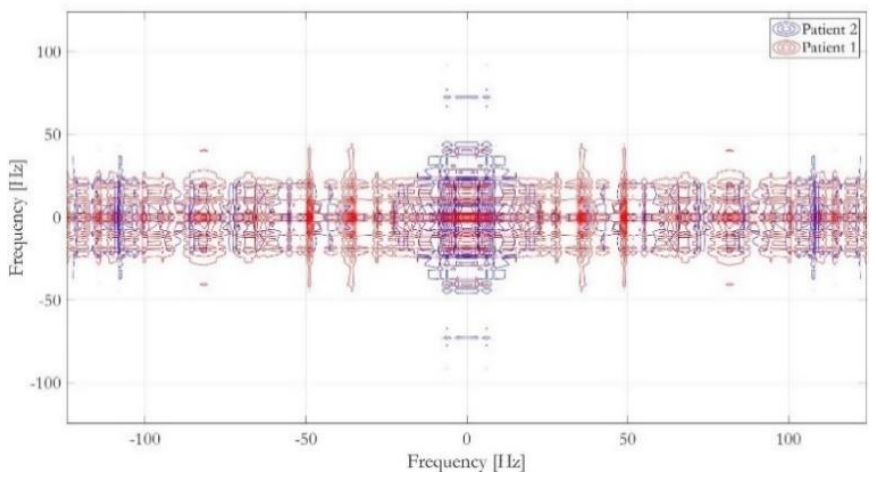

Figure 12. Superposition of bispectrums associate to patient 1 and patient 2 . 
The personalized care is another field of the unifying platform. The contributions of this research inevitably include data reduction and high-fidelity signal processing to a local level to extract characteristic features and communicate them to the cloud database. A demonstration of EEG and ECG features extraction was carried out, and detailed on how the deployment of obtained processing results on a cloud-based application has been presented. To obtain the desired outcome, the study suggested a deployment using a system consisting of at least $900 \mathrm{MHz} 32$ quad-core ARM Cortex-A7 CPU, and 2 GB RAM. The dataset exploited is from MIT-BIH Arrhythmia, and ranging from $16.24 \mathrm{kB}$ to $36.45 \mathrm{kB}$. The suggested architecture makes possible the reduction of data around $97 \%$ while most suggested architectures in the litteratre present an accuracy about $89 \%$. Moreover, the time taken by the transfert is estimate to 15 seconds, which validates the efficacy of the proposed architecture in monitoring vital signs such EEG and ECG in even real-time. On the other hand, the study provides a highlevel understanding of the cloud-bade IoT system and remote healthcare monitoring.

\section{REFERENCES}

[1] Boyi Xu, Lida Xu, Hongming Cai, Lihong Jiang, Yang Luo, Yizhi $\mathrm{Gu}$, The design of an $\mathrm{m}$-Health monitoring system based on a cloud computing platform, Enterprise Information Systems, vol. 11, issue 1, 2017, pp.17-36. DOI: $\underline{10.1080 / 17517575.2015 .1053416}$

[2] T. Han, L. Zhang, S. Pirbhulal, W. Wu, V. H. de Albuquerque, A novel cluster head selection technique for edge-computing based IoMT systems. Comput Network. April 2019, 158(2), 114e122. DOI: $10.1016 /$ j.comnet.2019.04.021

[3] B. Kamsu-Foguem, P. F. Tiako, L. P. Fosto, C. Foguem, Modeling for effective collaboration in telemedicine, Telematics and Informatics, vol. 32, issue 4, November 2015, pp. 776-786. DOI: $10.1016 /$ i.tele.2015.03.009

[4] A. Lay-Ekuakille, P. Vergallo, G. Griffo, F. Conversano, S. Casciaro, S. Urooj, V. Bhateja, A. Trabacca, Entropy Index in Quantitative EEG Measurement for Diagnosis Accuracy, IEEE Transactions on Instrumentation \& Measurement, vol. 63, n. 6, 2014, pp. $1440-1450$.

DOI: $\underline{10.1109 / T I M .2013 .2287803}$

[5] Weisong Shi, Jie Cao, Quan Zhang, Youhuizi Li, Lanyu Xu, Edge Computing: Vision and Challenges', IEEE Internet of Things Journal, vol. 3, issue 5, Oct. 2016, pp. 637 - 646. DOI: $10.1109 /$ JIOT.2016.2579198

[6] Cisco, Fog Computing and the Internet of Things: Extend the Cloud to Where the Things Are, White paper, 2015. Online [Accessed 13 September 2021]

https://www.cisco.com/go/iot

[7] Deepak Puthal, Saraju P. Mohanty, Uma Choppali, Collaborative Edge Computing for Smart Villages, IEEE Consumer Electronics Magazine, vol. 10, issue 3, 1 May 2021, pp. 68-71.

DOI: $10.1109 /$ MCE.2021.3051813

[8] Kai Wang, Hao Yin, Wei Quan, Geyong Min, Enabling Collaborative Edge Computing for Software Defined Vehicular Networks, IEEE Network, vol. 32, issue 5, September/October 2018, pp. 112-117.

DOI: $10.1109 / \mathrm{MNET} .2018 .1700364$

[9] X. Chen L. Jiao, W. Li, X. Fu, Efficient Multi-User Computation Offloading for Mobile-Edge Cloud Computing, IEEE/ACM Transactions on Networking, vol. 24, issue 5, October 2016, pp. 2795-2808.

DOI: $10.1109 /$ TNET.2015.2487344

[10] A. Saeed, M. Ammar, K. A. Harras, E. Zegura, Vision: The case for symbiosis in the Internet Of Things, Proc. 6th International Workshop on Mobile Cloud Computing and Services, Paris,
France, 11 September 2015, pp. 23-27.

DOI: $\underline{10.1145 / 2802130.2802133}$

[11] T. X. Tran, A. Hajisami, P. Pandey, D. Pompili, Collaborative mobile edge computing in 5G networks: New paradigms, scenarios, and challenges, IEEE Communications Magazine, vol. 55, issue 4, April 2017, pp. 54-61.

DOI: $\underline{10.1109 / M C O M .2017 .1600863}$

[12] A. Lay-Ekuakille, P. Vergallo, A. Trabacca, M. De Rinaldis, F. Angelillo, F. Conversano, S. Casciaro, Low-Frequency Detection in ECG Signals and Joint EEG-Ergospirometric Measurements for Precautionary Diagnosis, Measurement, vol. 46, issue 1, 2012, pp. $97-107$.

DOI: $10.1016 /$ i.measurement.2012.05.024

[13] K. Wang, H. Yin, W. Quan, G. Min, Enabling collaborative edge computing for software defined vehicular networks, IEEE Network, vol. 32, issue 5, Sep./Oct. 2018, pp. 112-117. DOI: $10.1109 /$ MNET.2018.1700364

[14] H. Zhang, P. Dong, W. Quan, B. Hu, Promoting efficient communications for high-speed railway using smart collaborative networking, IEEE Wireless Communications, vol. 22, issue 6, Dec. 2015, pp. 92- 97.

DOI: $10.1109 /$ MWC.2015.7368829

[15] L. Chen, J. Xu, Socially trusted collaborative edge computing in ultra dense networks, Proc. of the 2nd ACM/IEEE Symposium on Edge Computing, San Jose/Fremont, CA, USA, 12-14 October 2017, 11 pp. DOI: $\underline{10.1145 / 3132211.3134451}$

[16] Yuvraj Sahni, Jiannong Cao, Lei Yang, Data-aware task allocation for achieving low latency in collaborative edge computing, IEEE Internet of Things Journal, vol. 6, issue 2, April 2019, pp. 35123524.

DOI: $\underline{10.1109 / J I O T .2018 .2886757}$

[17] A. Lay-Ekuakille, S. Ikezawa, M. Mugnaini, R. Morello, Detection of Specific Macro and Micropollutants in Air Monitoring, Review of Methods and Techniques, Measurement, 98(1) (2017), pp. 4959. DOI: $10.1016 /$ i.measurement.2016.10.055

[18] A. P. Plonski, J. Vander Hook, V. Isler, Environment and Solar Map Construction for Solar-Powered Mobile Systems, IEEE Transactions on Robotics, vol. 32, issue 1, Feb. 2016, pp. 70-82. DOI: $\underline{10.1109 / T R O .2015 .2501924}$

[19] World Health Organization, Coronavirus disease (COVID-19) pandemic website. Online [Accessed 9 September 2021] https://www.who.int/emergencies/diseases/novel-coronavirus2019.

[20] A. Lay-Ekuakille, M. A. Ugwiri, C. Liguori, P. K. Mvemba, Proceedings of the Medical Measurements and Applications, (MeMeA) Symposium, Istanbul, Turkey, 26-28 June 2019, art. no. 8802127.

DOI: $10.1109 / \mathrm{MeMeA} .2019 .8802127$

[21] A. Lay-Ekuakille, G. Griffo, P. Visconti, P. Primiceri, R. Velazquez, Leaks Detection in Waterworks: Comparison between STFT and FFT with an Overcoming of Limitations, Metrology and Measurement Systems, vol. 24, issue 4, pp. 631 - 644. DOI: $10.1515 / \mathrm{mms}-2017-0049$

[22] B. Qureshi, Towards a digital ecosystem for predictive healthcare analytics, Proc. of MEDES 2014 - 6th International Conference on Management of Emergent Digital EcoSystems, Buraidah Al Qassim, Saudi Arabia, 15 - 17 September 2014, pp. 34-41. DOI: $10.1145 / 2668260.2668286$

[23] H. Patel, T. M. Damush, E. J. Miech, N. A. Rattray, H. A. Martin, A. Savoy, L. Plue, J. Anderson, S. Martini, G. D. Graham, L. S. Williams, Building cohesion in distributed telemedicine teams: findings from the Department of Veterans Affairs National Telestroke Program, BMC Health Services Research, 21 (1), art. no. 124.

DOI: $10.1186 / \mathrm{s} 12913-021-06123-\mathrm{x}$ 